\title{
Classificação de barragens de contenção de rejeitos de mineração e de resíduos industriais em relação a fatores de risco
}

\author{
(Risk-factor classification of tailings and industrial waste dams)
}

Terezinha de Jesus Espósito

Doutora, Professora Adjunta do Departamento de Engenharia de Transportes e Geotencia, Escola de Engenharia, Universidade Federal de Minas Gerais

E-mail: esposito@etg.ufmg.br

\section{Anderson Pires Duarte \\ Mestre em Meio Ambiente Departamento de Engenharia Sanitária e Ambiental, Universidade Federal de Minas Gerais E-mail: andersonpduarte@yahoo.com.br}

\section{Resumo}

Recentes acidentes envolvendo barragens de contenção de rejeitos de mineração e de resíduos industriais vêm despertando a atenção para essas estruturas, que crescem com o aumento da produção industrial. Em Minas Gerais, a Fundação Estadual do Meio Ambiente (FEAM) realiza cadastro e classificação dessas barragens através da metodologia de Potencial de Dano Ambiental. De acordo com essa classificação as barragens devem se submeter, periodicamente, a novas auditorias técnicas de segurança, com prazos de um, dois ou três anos. Em parceria com a FEAM, foram aplicados dados de 124 barragens de contenção de rejeitos de mineração e de resíduos industriais em um modelo de avaliação de potencial de risco, a princípio, desenvolvido para barragens convencionais. O modelo, que considera os fatores Periculosidade, Vulnerabilidade e Importância Estratégica, se apresenta como uma ferramenta adicional para subsidiar a tomada de decisões, permitindo focar atenção naquelas barragens que apresentarem situação mais crítica quanto ao potencial de risco e de dano ambiental. Os resultados mostraram que nenhuma barragem apresentou potencial de risco Muito Alto/Emergência. No entanto, 30\% das barragens apresentaram potencial de risco Médio/Alerta, o que revela a necessidade de priorizar a atenção e a intervenção nessas estruturas.

Palavras-chave: Rejeitos de mineração, resíduos industriais, segurança de barragens, classificação de barragens, potencial de risco, potencial de dano ambiental.

\section{Abstract}

Recent accidents involving tailings and industrial waste dams are attracting attention. This is because the number of these dams increases as industrial production increases. In Minas Gerais, the State Environmental Foundation (FEAM) conducts the registration and classification of dams according to the methodology called Potential Environmental Damage. According to this classification, the dams must be periodically submitted to technical and safety audits every one, two or three years. In partnership with FEAM, data from 124 tailings and industrial waste dam was applied in a model for potential-risk evaluation; in principle, developed for conventional dams. The model, which considers factors of danger, vulnerability and strategic importance, is presented as an additional tool to support decision-making, allowing focusing attention on those
\end{abstract}


dams that present the most critical risk potential for environmental damage. The results showed that no dam presented the Very High/Emergency potential risk. However, $30 \%$ of the dams showed Medium/Alert potential risk, which shows the need to prioritize attention and intervention in these structures.

Keywords: Tailings, industrial waste, dam safety, dam classification, risk potential, environmental damage potential.

\section{Introdução}

Existem, no Brasil, inúmeros barramentos de diversas dimensões e destinados a diferentes usos, tais como barragens de infra-estrutura para acumulação de água, geração de energia, aterros ou diques para retenção de resíduos industriais, barragens de contenção de rejeitos de mineração, entre outros. A diversidade de tamanhos e usos das barragens e aterros reflete-se, também, nas condições de manutenção dessas estruturas. Algumas são impecavelmente mantidas, atendendo normas de segurança compatíveis com os padrões internacionais mais exigentes, enquanto outras se apresentam com sérios riscos de serem ultrapassados os limites de segurança, podendo, inclusive, resultar no rompimento da estrutura.

Nesse artigo, são estudadas algumas barragens de contenção de rejeitos, assim como barragens para conteção de resíduos industriais. As barragens de contenção de rejeitos são estruturas construídas para conter os materiais produzidos, paralelamente ao produto final, no processo de beneficiamento do minério. Essas barragens são executadas em estágios, na medida em que esses rejeitos são gerados, diluindo, assim, os custos da construção e da operação. Tanto as barragens de contenção de rejeitos como as de resíduos industriais são reconhecidas por gerarem um impacto ambiental significante. Nesse sentido, a gestão dos rejeitos e resíduos está se tornando um dos critérios pelos quais o desempenho ambiental das empresas é julgado. Falhas ocorridas nessas barragens custaram vidas e causaram danos ambientais consideráveis. Acidentes graves resultaram em grandes volumes de rejeitos e resíduos descarregados no meio ambiente. Têm-se, também, os reservatórios, cada vez maiores, envolvendo, muitas vezes, efluentes tóxicos e outros materiais potencialmente perigosos. Uma razão comum para as falhas é que, muitas vezes, essas barragens não são operadas de acordo com critérios adequados para projeto, construção e operação.

Em Minas Gerais, a Fundação Estadual do Meio Ambiente (FEAM), após o acidente com a barragem da Mineração Rio Verde, ocorrido em 2001, iniciou um levantamento do número e do tipo de barragens existentes nas indústrias e nas minerações do Estado. Esse trabalho vem sendo realizado, tendo em vista o cadastramento e a classificação das barragens, seguindo parâmetros da Deliberação Normativa DN 62 (COPAM, 2002), que foi alterada pela DN 87 (COPAM, 2005) e pela DN 113 (COPAM, 2007). A classificação indicada, nessas deliberações, estabelece classes de potencial de dano ambiental (I, II e III), estimando as consequências em relação ao dano ambiental, em caso de uma ruptura. De acordo com essa classificação, devem ocorrer auditorias técnicas periódicas em prazos de um, dois ou três anos, para as barragens classificadas como III, II e I, respectivamente. Essas auditorias são realizadas por consultor externo ao quadro de funcionários da empresa. Os relatórios dessas auditorias têm como objetivos conhecer as condições físicas e estruturais das barragens e atestar se as mesmas apresentam condições de estabilidade. Caso não apresentem, o relatório deve conter um plano de ações, com prazo para implantação de melhorias, a fim de corrigir as falhas e garantir a estabilidade das estruturas.

Inserido no contexto da gestão da segurança dessas estrutras, esse artigo apresenta o resultado de uma pesquisa, que aplicou dados de 124 barragens de contenção de rejeitos de mineração e de resíduos industriais do Estado de Minas
Gerais em um modelo de potencial de risco, que se baseou na metodologia desenvolvida por Menescal et al. (2001). Essas barragens foram selecionadas por critérios de disponibilidade de dados para aplicação no modelo, função do reservatório (armazenamento de resíduos industriais ou rejeitos de mineração), classificação de dano ambiental e localização geográfica. O modelo teve, como objetivo, estimar o risco potencial de ruptura e definir prioridades de intervenção, alocação de recursos de manutenção e recuperação, bem como servir de referência para ações de segurança, priorização de investimentos em reparos, melhorias, monitoramento por instrumentação, periodicidade de inspeções, modernização de equipamentos, planos de ação emergenciais, entre outros (Duarte, 2008).

\section{Metodologia}

As informações apresentadas a seguir baseiam-se na disertação de mestrado desenvolvida por Duarte (2008). Dados de barragens de contenção de rejeitos de mineração e de resíduos industriais do Estado de Minas Gerais foram aplicados em um modelo de avaliação de potencial de risco, que considera os fatores Periculosidade, Vulnerabilidade e Importância Estratégica. Para o preenchimento desse modelo, foram utilizados dados secundários, num trabalho realizado em parceria com a FEAM.

Numa primeira etapa, foram analisados os relatórios das 606 barragens cadastradas pela FEAM em 2006 e verificados quais seriam aplicáveis a essa pesquisa. Após essa etapa, foram selecionados 124 relatórios de auditoria técnica de barragens, com informações completas, sendo esse grupo considerado o espaço amostral.

Foi identificada a necessidade de realizar alterações no modelo proposto por Menescal et al. (2001), adaptandoo, de modo a torná-lo aplicável às barragens de contenção de rejeitos de mineração e de resíduos industriais. Dessa forma, os dados das 124 barra- 
gens selecionadas foram aplicados ao modelo, que, após as alterações, passou a ser chamado de modelo Potencial de Risco Modificado.

A fase seguinte foi a calibração do modelo, para garantir que a pontuação estabelecida, para cada classe, fosse realmente representativa para definição do potencial de risco das barragens. Para isso, foi utilizado o próprio banco de dados do grupo em que algumas barragens já apresentavam estabilidade garantida pelo auditor, enquanto outras apresentavam problemas de estabilidade. As barragens que não apresentavam estabilidade garantida pelo auditor não foram aplicadas na pesquisa, mas funcionaram como Grupo Controle para calibração do modelo.

Assim, as barragens envolvidas foram divididas em 2 (dois) grupos:

- Grupo 1: 101 barragens de contenção de rejeitos de mineração e de resíduos industriais. Os dados desse grupo foram aplicados no modelo de Potencial de Risco Modificado.

- Grupo Controle: 23 barragens que, de acordo com os relatórios de auditorias técnicas, não possuíam estabilidade garantida pelo auditor. Esse grupo funcionou como controle dos resultados e calibração da pontuação do modelo.

Os resultados obtidos pela aplicação dos dados das 101 barragens no modelo de Potencial de Risco Modificado foram comparados com a classificação atual de Potencial de Dano Ambiental estabelecida pela DN 87 (COPAM, 2005).

\section{Resultados}

Foi calculado o Potencial de Risco de todas as barragens, a partir da determinação dos valores dos fatores Periculosidade, Vulnerabilidade e Importância Estratégica, atribuindo-se uma pontuação a todos os parâmetros que compõem cada fator.

\subsection{Periculosidade}

A periculosidade $(\mathrm{P})$ representa as características técnicas da barragem, sendo que o conjunto dos seus parâmetros indica se o estado atual da barragem oferece algum perigo. A referida periculosidade é obtida pela soma das pontuações, indicadas pela metodologia, dos parâmetros altura, volume, tipo de barragem, tipo de fundação e vazão de projeto, conforme equação 1 (Duarte, 2008):

$P=\sum_{1}^{5} p i$

$P$ e $p$ representam, respectivamente, a periculosidade e o peso de cada parâmetro. Se $P$ for maior do que 30, a periculosidade é considerada elevada; se estiver entre 20 e 30, é significativa e, se estiver entre 10 e 20, é considerada baixa a moderada. A Figura 1 apresenta as porcentagens encontradas para cada nível de periculosidade.

\subsection{Vulnerabilidade}

A vulnerabilidade (V) representa a situação atual da barragem, procurando medir o quanto as estruturas estão vulneráveis a uma possível ruptura. Busca, ainda, mensurar a extensão dos danos materiais e financeiros daí advindos. A referida vulnerabilidade é obtida através da soma das pontuações, indicadas pela metodologia, atribuídas aos parâmetros idade, existência de projeto As Built, confiabilidade das estruturas vertedouras, alteamento, percolação, presença de deformações ou afundamentos, deterioração dos taludes e instrumentação e monitoramento, conforme equação 2 (Duarte, 2008):

$V=\sum_{6}^{13} v i$

$V$ e $v$ representam, respectivamente, a vulnerabilidade e o peso de cada parâmetro. Se o valor de V for maior do que 40, tem-se vulnerabilidade elevada; se V estiver entre 22 e 40, vulnerabilidade moderada a elevada; V entre 5 e 22, vulnerabilidade baixa a moderada e $\mathrm{V}$ maior do que 5, vulnerabilidade muito baixa. A Figura 2 apresenta as porcentagens encontradas para cada nível de vulnerabilidade.

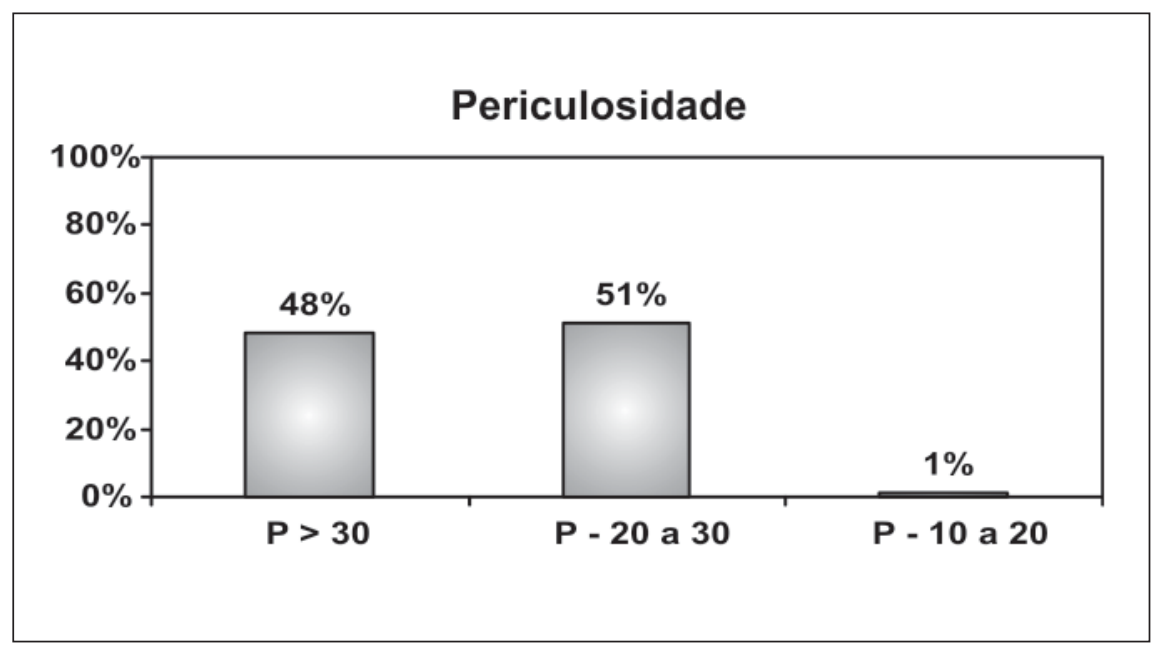

Figura 1 - Periculosidade. Fonte: Duarte (2008). 


\subsection{Importância estratégica}

A importância estratégica (I) representa a dimensão dos possíveis impactos (econômicos e ambientais) em caso da ruptura de uma barragem. É obtida através da média aritmética do volume atual (A), da população a jusante (B) e do interesse econômico e ambiental da área a jusante (C), através da Equação 3 (Duarte, 2008). A Tabela 1 apresenta os valores atribuídos a cada parâmetro. As Figuras 3, 4 e 5 apresentam as porcentagens encontradas para cada item da importância estratégica.

$I=\frac{A+B+C}{3}$

Os valores adotados, em relação ao item População a Jusante (B), foram os mesmos relativos ao parâmetro "Ocupação Humana a Jusante”, utilizado na classificação quanto ao Potencial de Dano Ambiental realizada pela FEAM (2007), de acordo com a DN 87 (COPAM, 2005). Em relação ao item Interesse Econômico e Ambiental (C), foi realizada uma compilação de informações sobre "Interesse Ambiental a Jusante” e "Instalações na Área de Jusante”, presentes no cadastramento das barragens, realizado pela FEAM (2007), utilizando, também, os critérios da DN 87 (COPAM, 2005).

\subsection{Resultados obtidos para o potencial de risco}

Com os valores calculados para os três fatores, ou seja, Periculosidade (P), Vulnerabilidade (V) e Importância Estratégica (I), foi obtido o Potencial de Risco (PR), para cada barragem, conforme Equação 4.

$P R=\frac{\left(\sum P+\sum V\right)}{2} \times I$

As barragens foram classificadas, de acordo com os valores do Potencial de Risco (PR) calculados, em níveis Alto/ Emergência, Médio/Alerta, Normal, Baixo ou Muito Baixo, conforme Tabela 2. Os resultados estão apresentados na Figura 6.

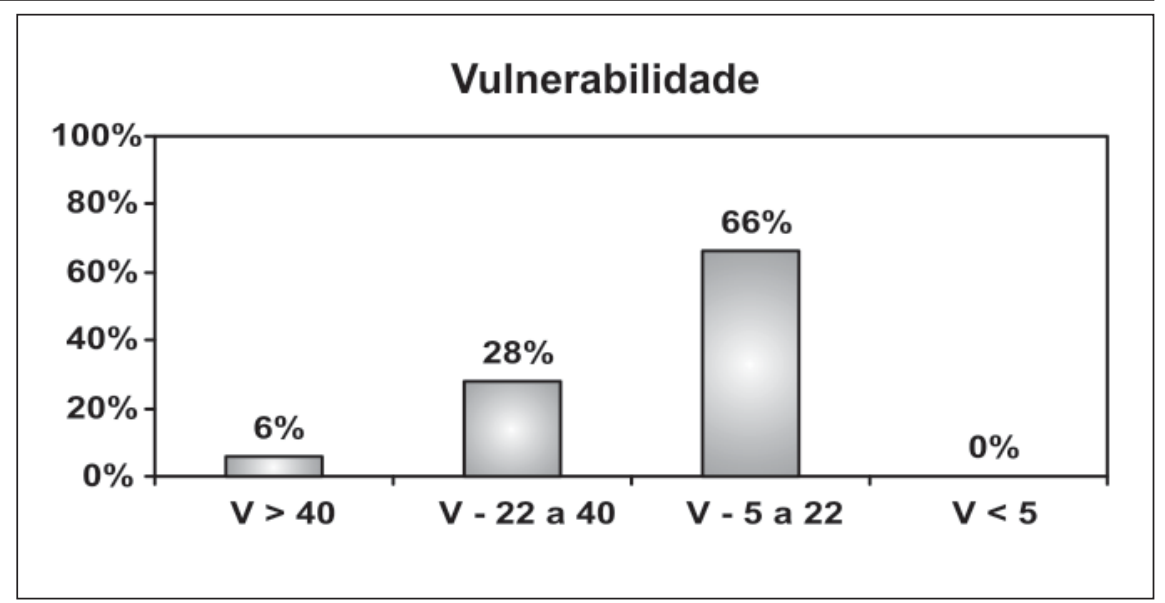

Figura 2 - Vulnerabilidade. Fonte: Duarte (2008).

Tabela 1 - Importância Estratégica (I): valores associados a cada parâmetro. Fonte: Duarte (2008).

\begin{tabular}{c|c|c|c|c|c}
\hline $\begin{array}{c}\text { Volume Atual } \\
\text { (A) }\end{array}$ & Valor & $\begin{array}{c}\text { População } \\
\text { a Jusante } \\
\text { (B) }\end{array}$ & Valor & $\begin{array}{c}\text { Interesse } \\
\text { Econômico e } \\
\text { Ambiental (C) }\end{array}$ & Valor \\
\hline Grande > $20 \mathrm{hm}^{3}$ & 2,0 & Grande & 2,50 & Elevado & 1,5 \\
\hline Médio 5 a $20 \mathrm{hm}^{3}$ & 1,5 & Média & 2,00 & Médio & 1,2 \\
\hline Baixo $<5 \mathrm{hm}^{3}$ & 1,0 & Pequena & 1,00 & Pequeno & 1,0 \\
\hline
\end{tabular}

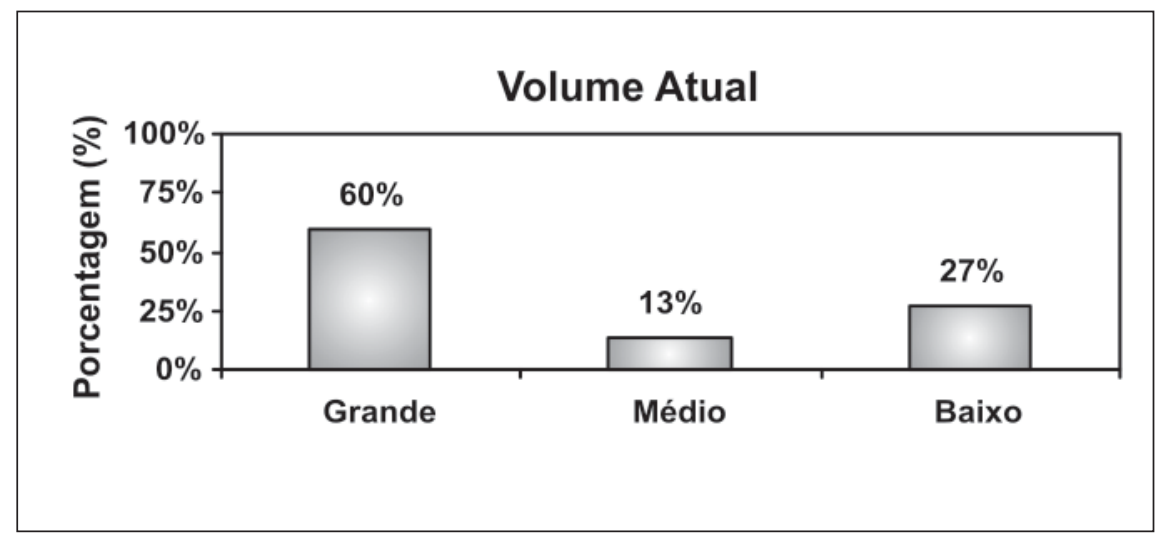

Figura 3 - Importância estratégica: volume atual. Fonte: Duarte (2008).

\section{Discussão}

Os resultados obtidos pelo modelo Potencial de Risco Modificado - PRM (Duarte, 2008) foram comparados com os gerados através da metodologia de Potencial de Dano Ambiental PDA (FEAM, 2007). Ao se fazer essa comparação, é relevante destacar que um modelo avalia o potencial de risco de ruptura, enquanto o outro avalia as consequências em relação ao dano ambiental, em caso de uma ruptura. Outro aspecto a ser salientado é que a metodologia PDA classifica as estruturas 
em 3 classes (I, II e III), enquanto que a metodologia PRM assume 5 classes (Alto/Emergência, Médio/Alerta, Normal, Baixo ou Muito Baixo). Entretanto nenhuma barragem do universo avaliado se enquadrou nas classes extremas do modelo PRM, ou seja, Muito Baixo e Alto/Emergência. Dessa forma, se, apenas para fins de comparação, fosse adotado o mesmo número de classes, nos dois modelos, desconsiderando-se as classes extremas do modelo PRM, poder-se-ia realizar uma analogia entre as metodologias. A Tabela 3 apresenta os resultados dos modelos Potencial de Risco Modificado (PRM) e Potencial de Dano Ambiental (PDA).

Vale destacar que as porcentagens de barragens classificadas pelo PRM como "Baixo" e pelo PDA como "Classe I” (Baixo) são muito próximas (17 \% e $16 \%$, respectivamente). No entanto, a partir da classificação "Normal” do PRM e “Classe II” (Médio), do PDA, esse quadro se inverte. Uma justificativa poderia estar associada aos parâmetros avaliados em cada metodologia. O modelo PRM apresenta parâmetros que não são considerados na DN 87 (COPAM, 2005) e que são significativos para definição dos resultados. O modelo apresentado, nesse artigo (PRM), incorpora parâmetros específicos dessas estruturas, para a avaliação do potencial de risco de ruptura.

\section{Conclusões}

Barragens de maiores dimensões, com mais disponibilidade de informações, desde o projeto até o programa de monitoramento, se apresentaram mais viáveis para a aplicação do modelo Potencial de Risco Modificado. Entretanto, se os órgãos fiscalizadores estabelecerem, nos próprios relatórios de auditorias técnicas de segurança, especificações de informações mais compatíveis com as necessidades dos dados necessários a esse modelo, essa ausência de dados, em barragens de menor porte, é passível de ser resolvida. Dentro desse contexto, foi observado, também, que, para as barragens que apresentaram relatórios de auditorias técnicas mais completos,

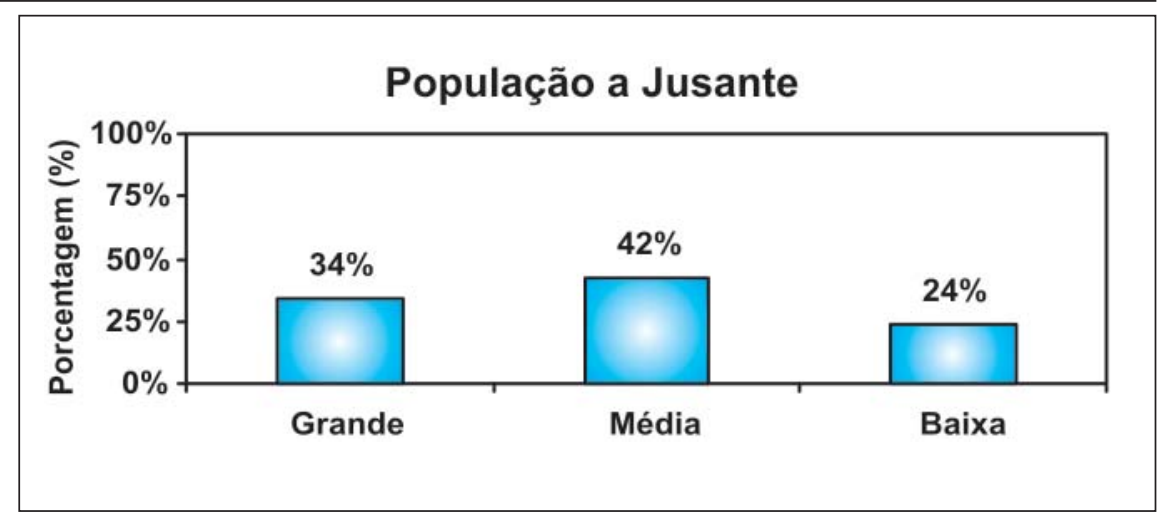

Figura 4 - Importância estratégica: população a jusante. Fonte: Duarte (2008).

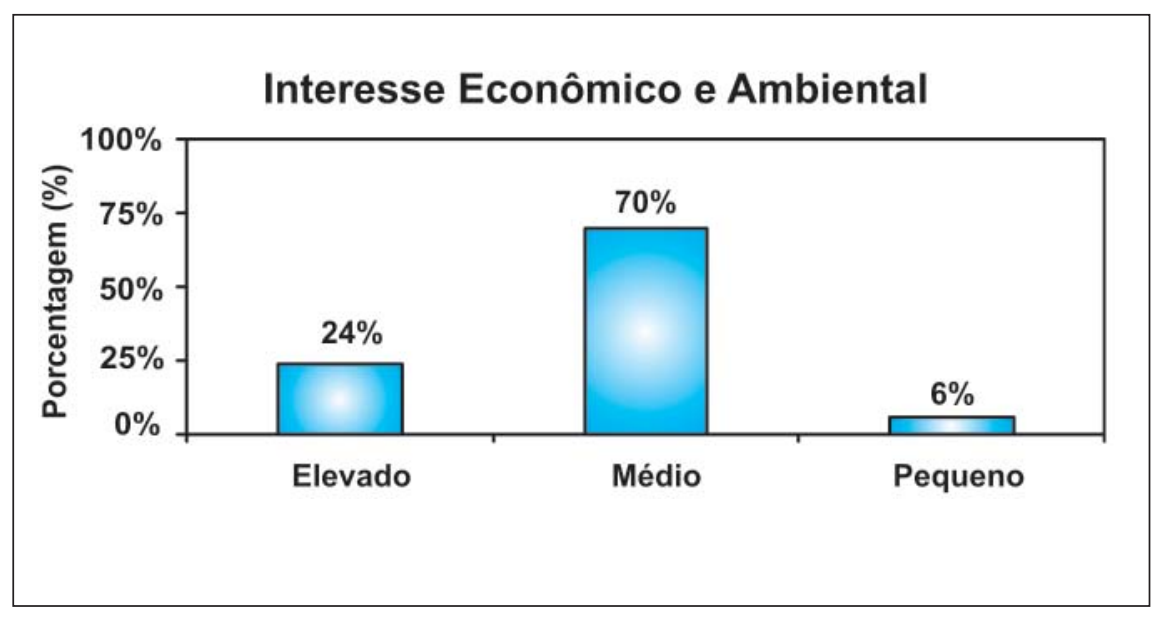

Figura 5 - Importância estratégica: interesse econômico e ambiental. Fonte: Duarte (2008).

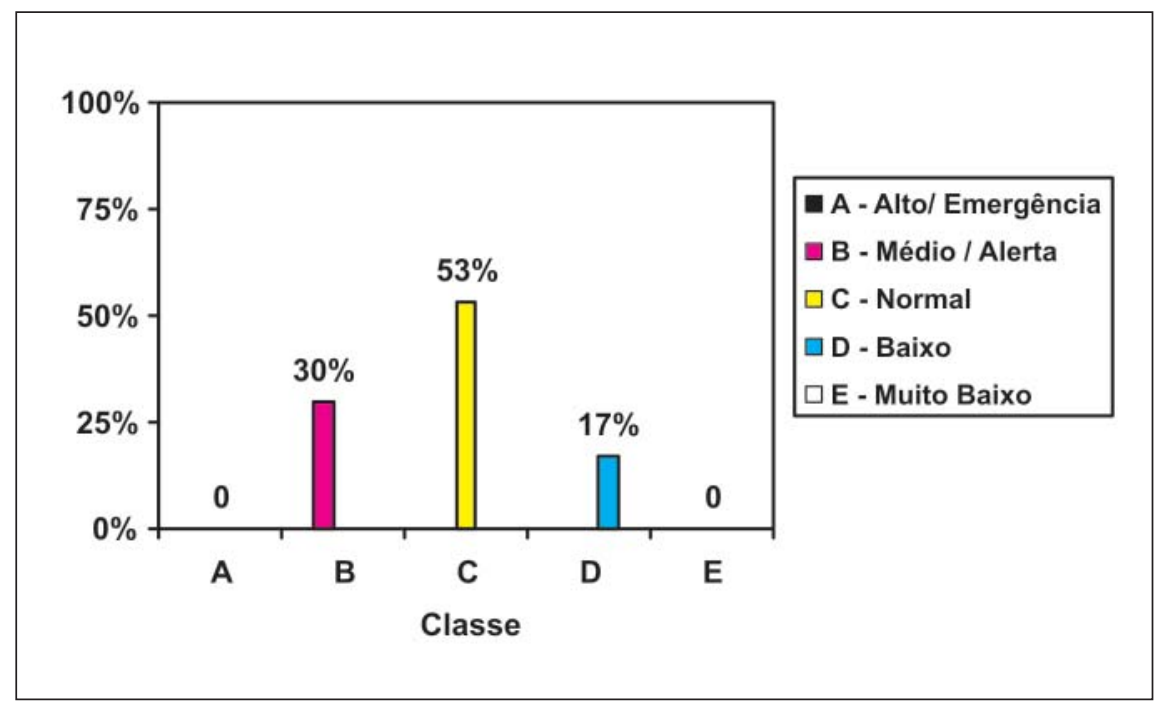

Figura 6 - Classificação segundo o modelo potencial de risco modificado. Fonte: Duarte (2008). 
Classificação de barragens de contenção de rejeitos de mineração e de resíduos industriais em ...

o preenchimento do modelo foi mais eficaz, o que leva a concluir que uma padronização desses relatórios poderia vir a contribuir para uma melhor geração de dados.

Outro aspecto a ser considerado é que os instrumentos de política ambiental podem conduzir a processos, por vezes, complexos e burocráticos. Dessa forma, ferramentas para gestão de segurança surgem como grande valia para manutenção dos empreendimentos, na conciliação de interesses econômicos e ambientais. Pode-se dizer, portanto, que, juntamente com a classificação de dano ambiental, estabelecida pela DN 87 (COPAM, 2005), os empreendedores e órgãos fiscalizadores possuem em mãos mais uma ferramenta de gestão, que permite intervenção de forma preventiva, possibilitando antecipação de ações e evitando eventos inesperados. Nesse sentido, a metodologia de análise do Potencial de Risco Modificada se mostrou como uma ferramenta eficaz, que muito pode contribuir no entendimento do comportamento de uma barragem, definindo pontos para inspeção estratégica, identificando medidas de redução de risco e priorizando as investigações e apoio nas tomadas de decisões.

Sugere-se, entretanto, que sejam também realizados estudos para a redução das incertezas em relação ao potencial de risco, considerando os diversos modos de ruptura, tais como galgamento por cheias, instabilidade de taludes e ruptura interna (por piping e sismos), além da aplicação de metodologias de análise de riscos.

\section{Agradecimentos}

Os autores agradecem à FEAM, por permitir a realização desse estudo e pela disponibilidade de informações utilizadas e publicadas nesse artigo.
Tabela 2 - Pontuação do modelo Potencial de Risco Modificado. Fonte: Modificado de Menescal et al. (2001) apud Duarte (2008).

\begin{tabular}{c|c}
\hline Classe & Potencial de Risco - PR \\
\hline A & $>70$ - Alto/Emergência \\
\hline B & 46 a 70 - Médio/Alerta \\
\hline C & 28 a $45-$ Normal \\
\hline D & 16 a 27 - Baixo \\
\hline E & $<15$ - Muito Baixo \\
\hline
\end{tabular}

Tabela 3 - Resultados dos modelos PRM e PDA. Fonte: Duarte (2008).

\begin{tabular}{c|c|c|c}
\hline $\begin{array}{c}\text { Potencial de Risco Modificado } \\
\text { (Duarte, 2008) }\end{array}$ & $\begin{array}{c}\text { Potencial de Dano Ambiental } \\
\text { (FEAM, 2007) }\end{array}$ \\
\hline Baixo & $17 \%$ & Classe I - Baixo & $16 \%$ \\
\hline Normal & $53 \%$ & Classe II - Médio & $29 \%$ \\
\hline Médio/ Alerta & $30 \%$ & Classe III - Alto & $55 \%$ \\
\hline
\end{tabular}

\section{Referências bibliográficas}

CONSELHO DE POLÍTICA AMBIENTAL - COPAM. Deliberação Normativa $N^{\circ}$ 62. Dispõe sobre critérios de classificação de barragens de contenção de rejeitos, de resíduos e de reservatório de água em empreendimentos industriais e de mineração no Estado de Minas Gerais. Lex: Diário do Executivo - Minas Gerais, 17 de dezembro de 2002. 9 p.

CONSELHO DE POLÍTICA AMBIENTAL - COPAM. Deliberação Normativa $N^{\circ}$ 87. Altera e complementa a Deliberação Normativa COPAM no 62, de 17/12/2002. Lex: Diário do Executivo - Minas Gerais, 18 de junho de 2005. 9 p.

CONSELHO DE POLÍTICA AMBIENTAL - COPAM. Deliberação Normativa $N^{\circ}$ 113. Altera e complementa a Deliberação Normativa COPAM no 62, de 17/12/2002. Estabelece prazo para apresentação de declaração sobre a situação das barragens. Lex: Diário do Executivo - Minas Gerais, 1 de dezembro de 2007. 1 p.

DUARTE, A. P. Classificação das barragens de contenção de rejeitos de mineração e de resíduos de contenção de rejeitos de mineração e de resíduos industriais no Estado de Minas gerais em relação ao potencial de risco. Belo Horizonte: Universidade Federal de Minas Gerais, 2008. 130 p. (Dissertação de Mestrado).

FUNDAÇÃO ESTADUAL DO MEIO AMBIENTE - FEAM. Evolução das ações de gestão de barragens de contenção de rejeitos, de resíduos e de reservatórios de água em empreendimentos industriais e minerários no Estado de Minas Gerais. Março 2007. 128 p. Disponível em: <http://www.feam.br>. Acesso em: 12 abr. 2007.

MENESCAL, R. A., CRUZ, P. T., CARVALHO, R. V., FONTENELLE, A. S., OLIVEIRA, S. K. F. Uma metodologia para avaliação do potencial de risco em barragens do semi-árido. In: SEMINÁRIO NACIONAL DE GRANDES BARRAGENS, 2001.

Artigo recebido em 26/01/2009 e aprovado em 04/09/2009. 